\title{
PTEN transcript variants caused by illegitimate splicing in "aged" blood samples and EBV-transformed cell lines
}

\author{
Yunying Liu · Priyangi Malaviarachchi · \\ Marjorie Beggs · Peter D. Emanuel
}

Received: 8 June 2010 / Accepted: 29 August 2010 / Published online: 14 September 2010

(C) The Author(s) 2010. This article is published with open access at Springerlink.com

\begin{abstract}
PTEN is one of the most frequently mutated tumor suppressor genes in human cancers. Mutations occur in either heritable or sporadic fashion. Sequencing of cDNA from patients and normal individuals often reveals splicing variants (SVs) of PTEN, some of which are nonmutation related. To investigate whether these SVs were the result of illegitimate splicing (a general decrease of fidelity in splicing site selection in "aged" samples), we tested "aged" blood from individuals who had normal PTEN transcripts in their "fresh" mononuclear cells. Blood from 20 normal individuals was collected and split into two aliquots. Total RNA and DNA were extracted immediately ("fresh") and $48 \mathrm{~h}$ later ("aged"), respectively. Using RT-PCR, subcloning and sequencing, we found seven types of SVs. No mutation was detected in the related intron-exon flanking region in genomic DNA in either "fresh" or "aged" samples. Some of the SVs were also consistently present in both the "fresh" and "aged" EBV-transformed lymphoblastoid cells from six normal individuals. Western blot data indicated that the PTEN protein level (in full length) was not altered in the "fresh" EBV-transformed lymphoblastoid cells with SVs. In conclusion, our data demonstrate that PTEN illegitimate splicing often occurs in "aged" blood and EBV-transformed lymphoblastoid cells. Therefore, it is critical to note the time point of RNA extraction when investigating for PTEN aberrant transcripts. We hope that our data will increase awareness about the sample status, because gene expression data may
\end{abstract}

Y. Liu · P. Malaviarachchi · M. Beggs · P. D. Emanuel $(\square)$

Winthrop P. Rockefeller Cancer Institute,

University of Arkansas for Medical Sciences,

Little Rock, AR, USA

e-mail: pdemanuel@uams.edu be potentially flawed from "aged" samples, particularly when dealing with clinical samples.

\section{Introduction}

PTEN (phosphatase and tensin homolog deleted on chromosome ten)/MMAC1 (mutated in multiple advanced cancers)/TEP1 (TGF- $\beta$-regulated and epithelial cell-enriched phosphatase, referred hereafter as $P T E N$ ), as a tumor suppressor, is a central negative regulator of the PI3K/AKT signaling cascade that influences multiple cellular functions including cell growth, survival, proliferation, and migration in a context-dependent manner (Carracedo and Pandolfi 2008). Germline mutations of PTEN (DNA Accession\# AF067844, mRNA Accession\# U92436, or U93051) cause the inherited Cowden Syndrome (CS; MIM 158350) and Bannayan-Riley-Ruvalcaba Syndrome (BRRS; MIM 153480) (Liaw et al. 1997; Marsh et al. 1998; Zhou et al. 2003). Somatic intragenic mutations or deletions of PTEN have been found in a wide variety of advanced sporadic tumors in humans, such as brain, breast, prostate, lung, ovary, thyroid, melanoma, endometrial, acute leukemia, and non-Hodgkin's lymphoma (Dahia et al. 1997, 1999; Kurose et al. 2001; Soria et al. 2002; Tashiro et al. 1997; Wang et al. 1997; Zhou et al. 2003). We recently reported that PTEN protein is also deficient in juvenile myelomonocytic leukemia (JMML) (Liu et al. 2009). Data from primary human tumors, as well as animal models, support the concept that the impact of PTEN inactivation is tissuespecific. For many years, researchers have been struggling to identify the mechanism that causes the deficiency in PTEN function. Some studies have demonstrated intriguing discontinuities between PTEN mutations and the subsequent phenotypes. Several groups have reported splice 
variants on PTEN in normal individuals (Sharrard and Maitland 2000), patients with inherited diseases or cancer (Agrawal and Eng 2006; Celebi et al. 2000), and tumor cell lines (Sharrard and Maitland 2000). However, only a few of them are mutation-associated (Celebi et al. 2000).

In the process of screening for PTEN mutations in patients with JMML, we found several aberrantly spliced transcripts, which were not related to any mutation at either the splice-donor and receptor-site, nor branch site. Alternative splicing is an important mechanism to create protein diversity and regulate gene expression in a tissue- and developmental-specific manner, and it plays an important role in cancer development (for more background see reviews by Caceres and Kalnina) (Caceres and Kornblihtt 2002; Kalnina et al. 2005). Studying alternative splicing has been very challenging due to the poor understanding of the mechanism of splicing regulation and the complexity of tissue status, which makes it very difficult to interpret data. In order to exclude that the SVs were not the result of illegitimate splicing (defined as a general decrease of fidelity in selection of splicing site in "aged" samples), a study was conducted on peripheral blood and Epstein-Barr virus (EBV)-transformed lymphoblastoid cells from normal individuals who did not have any aberrant splicing in mononuclear cells (MNCs) from their "fresh" blood samples.

\section{Methods}

Sample preparation

Blood from normal individuals was collected into heparinized tubes and split into two aliquots. MNCs were isolated immediately ("fresh") from one aliquot following the procedure previously reported (Liu et al. 2009). The second aliquot was kept at room temperature and the MNCs were isolated after $48 \mathrm{~h}$ ("aged"). Permission to use samples of normal individuals was granted by the Institutional Review Boards (IRB) at the University of Alabama at Birmingham and University of Arkansas for Medical Sciences.

\section{EBV lymphoblastoid cell lines}

Epstein-Barr virus-transformed lymphoblastoid cell lines from healthy individuals were established following the modified protocol by Glick (Glick 1980). Briefly, infectious EBV was obtained from 10-day-old cell-free supernatant of the virus producer B95-8 cell line (ATCC, Manassas, VA). MNCs from "fresh" blood were transformed in a density of $10 \times 10^{6} / \mathrm{ml}$ in $2 \mathrm{ml}$ of EBV virus supernatant for 3-6 h in $5 \% \mathrm{CO}_{2}$ at $37^{\circ} \mathrm{C}$, then the culture volume was expanded by adding RPMI-1640 medium (containing 20\% FBS plus penicillin and streptomycin) and cyclosporine A (Sigma-
Aldrich, St. Louis, MO) in a final concentration of $0.5 \mu \mathrm{g} / \mathrm{ml}$ to dilute the final cell density to $1 \times 10^{6} / \mathrm{ml}$. The cultures were then incubated in $5 \% \mathrm{CO}_{2}$ at $37^{\circ} \mathrm{C}$ for $5-8$ weeks until the cells were transformed and proliferating (watched for signs of gross clumping). Cells were frozen in 10\% DMSO in $100 \%$ FBS and stored in liquid nitrogen for future analysis.

\section{Extraction of DNA and RNA}

Mononuclear cells were lysed in Trizol reagent ${ }^{\circledR}$ (Invitrogen, Carlsbad, CA) immediately after being isolated. In order to prevent RNA degradation, EBV cell lines were pre-treated with puromycin $(150 \mu \mathrm{g} / \mathrm{ml})$ for $6 \mathrm{~h}$ before cell lysis. Total RNA and DNA were extracted from cell lysates according to the manufacturer's instruction, and quantified spectrophotometrically.

\section{RNA integrity analysis}

To determine the quality and quantity of RNA, one microliter of sample was analyzed using the RNA 6000 Nano Chip kit and the Agilent 2100 Bioanalyzer (Agilent Technologies, Inc, Santa Clara, CA). Concentrations are determined as nanogram per microliter and the integrity of the RNA is scored with an RNA integrity number (RIN) that grades RNA integrity from 0 (decayed) to 10 (intact).

\section{RT-PCR and sequence analysis}

Genomic DNA was removed from RNA by using the DNA-free kit (Ambion ${ }^{\circledR}$, Austin, TX) in order to prevent an amplification of the PTEN pseudogene. Two hundred twenty nanograms of total RNA were used as templates to synthesize first strand cDNA, in a total volume of $100 \mu \mathrm{l}$ by using the High-Capacity cDNA Reverse Transcription Kit (Applied Biosystems, Foster City, CA). The first-run PCR was amplified using GeneAmp PCR System 2700 (PerkinElmer Corp., Branchbury, NJ) and Platinum ${ }^{\circledR}$ Taq DNA polymerase high fidelity kit (Invitrogen). A $50 \mu$ of total volume of PCR reaction contained 1-5 $\mu \mathrm{l}$ of template, $5 \mu \mathrm{l}$ of $10 \times$ high fidelity PCR buffer, $2 \mathrm{mM} \mathrm{MgSO} 2,250 \mu \mathrm{M}$ of each dNTPs, 1 unit Platinum ${ }^{\circledR}$ Taq DNA polymerase high fidelity, and 20 pmol of forward and reverse primers. PTEN primer sequences are listed in Table 1. Amplification employed 35 cycles of the following sequence: denaturation at $94^{\circ} \mathrm{C}$ for $10 \mathrm{~s}$, annealing at $54-58^{\circ} \mathrm{C}$ for $30-60 \mathrm{~s}$, and elongation at $68^{\circ} \mathrm{C}$ for $90 \mathrm{~s}$. Primers $(1005 \mathrm{~F} / 2260 \mathrm{R})$ for the full length of $P T E N$-coding sequences were used in the first-run PCR. For sequencing, a pair of nested primers (92436F/871R) was used. For SVs screening, a pair of exon-specific primers was used in a second run PCR for each exon and flanking region (92436F/225R for intron-3 
Table 1 List of primers used in RT-PCR

\begin{tabular}{lll}
\hline Primer ID & Sequence & Location \\
\hline $1005 \mathrm{~F}$ & TCCTTTTTCTTCAGCCACAG & 5'UTR $^{\prime}$ \\
$2260 \mathrm{R}$ & TTCATGGTGTTTTATCCCTCTT & $3^{\prime} \mathrm{UTR}$ \\
$92439 \mathrm{~F}$ & GACAGCCATCATCAAAGAGA & Exon 1 \\
$871 \mathrm{R}$ & GATCACATAGACTTCCATTTTC & Exon 8 \\
$225 \mathrm{R}$ & TAAATTTGGCGGTGTCATAA & Exon 4 \\
$324 \mathrm{~F}$ & TGACCAATGGCTAAGTGAAG & Exon 4 \\
$501 \mathrm{R}$ & CGCCTCTGACTGGGAATA & Exon 6 \\
$674 \mathrm{R}$ & GGTCCTGAATTGGAGGAAT & Exon 7 \\
\hline
\end{tabular}

insertion, 324F/501R for intron-5 insertion, 324F/647R for exon-6 deletion). In order to ensure that there was no contamination by genomic DNA of the PTEN pseudogene, the same amount of total RNA (without being reverse-transcribed) from randomly selected control samples was amplified with the identical primers. Amplified PCR products were analyzed using electrophoresis with a $1.0-1.5 \%$ agarose gel stained with $0.5 \mu \mathrm{g} / \mathrm{ml}$ ethidium bromide. Products of nested PCR were amplified and purified by using TA Cloning Kit (Invitrogen) following the manufacture's instruction. Plasmid DNAs were purified using a miniPrep kit (Qiagen, Valencia, CA). Sequencing of purified products was performed using an ABI 373A DNA sequencer (Applied Biosystems). The sequence was analyzed using DNASIS-Mac v2.0 software.

\section{Western blot}

Unstimulated EBV lymphoblastoid cells from normal individuals were used for cell lysates. Western blots were performed as previously reported (Liu et al. 2009). Briefly, $25 \mu \mathrm{g}$ of cell lysate protein were separated in 10\% SDSpolyacrylamide gel (SDS-PAGE). Protein bands were electro-transferred onto a polyvinylidene difluoride (PVDF) membrane, and blocked in 5\% BSA/TBST $(20 \mathrm{mM}$ Tris$\mathrm{HCl}, \mathrm{pH} 7.6,150 \mathrm{mM} \mathrm{NaCl}, 0.1 \%$ Tween-20) for $1 \mathrm{~h}$ in room temperature. An antibody against human PTEN in rabbit antiserum (Upstate, Lake Placid, NY) was hybridized with the membrane at a concentration of 1:1,000 in 5\% BSA-TBST overnight at $4{ }^{\circ} \mathrm{C}$. The protein bands were detected by incubating the blot with 1:15,000 dilution of horseradish peroxide-conjugated anti-rabbit immunoglobin (Amersham Pharmacia biotech, Amersham Place, UK) for $1 \mathrm{~h}$ in room temperature, and then the blot was developed with ECL plus (Amersham Pharmacia Biotech) according to the manufacturer's instructions. The densities of protein bands were documented with Kodak XAR films. The same blot was further stripped with Restore ${ }^{\mathrm{TM}}$ Western Blot
Stripping Buffer (Pierce, Rockford, IL) at room temperature for $30 \mathrm{~min}$, then re-blocked, and re-probed with 1:5,000 dilution of $\beta$-actin (Sigma Chemical Co.) to normalize for equal protein loading for each sample.

Statistical calculation

The difference of RINs between "old" and "fresh" samples was evaluated using the student $t$ test. A $p$ value $<0.05$ $(p<0.05)$ was considered as statistically significant.

\section{Results}

PTEN splicing variants (SVs) are found in 'aged' blood from normal individuals but not in "fresh" blood from the same individuals

We initially drew blood from three healthy individuals. RT-PCR and sequencing demonstrated that seven types of SVs were found only in the "aged" blood samples, which were kept at room temperature for $48 \mathrm{~h}$ before MNCs were isolated, but were not found in their "fresh" blood counterparts. They included inclusions of portions of intron 3 , intron 5 , intron 1 , deletion of exon 6 , partial deletion of exon 8 , and a complete deletion of exon $2-5$. The intron inclusions occurred either alone or combined with exon(s) deletion (Fig. 1). There was no obvious connection indicating how the combinations would occur. In order to confirm this observation, we further collected peripheral blood from additional 17 normal individuals. By using exon-specific primers, we found these SVs are common in "aged" samples (Fig. 2). Four of the variants were previously reported by other groups, including retention of a portion of intron 3 immediately adjoined to the 3 ' of exon 3 ( $3 b$ and $3 c$ ) alone; inclusion of partial intron 5 (5c); and exon 6 skipping (DelE6), respectively (Agrawal and Eng 2006; Sharrard and Maitland 2000). No mutation was detected in the related intron-exon flanking region in genomic DNA in either "fresh" or "aged" blood (data not shown). Since the primers that were used in the first run of PCR amplified the full length of coding transcript of PTEN, we believe that these SVs were not due to RNA degradation. By searching the web site, http://www. fruitfly.org/sequence/humandatasets.html, analysis of the sequences of intron-3 and intron-5 retentions revealed that the inserted sequences were related to the putatively alternative splicing sites (scored as 0.23-0.97). This indicated that these SVs were not mutation-related; instead, most likely, they were caused by the relaxation of the splice fidelity when the samples were under stress. 
Fig. 1 Schema. This schema represents the identified splicing variants in "aged" blood and EBV-transformed lymphoblasts from healthy individuals. Genomic DNA sequence refers to Gene Bank/AF067844, mRNA refers to Gene Bank/U93051. The nomenclature used to describe the splice variants is according to the publication reported by Agrawal and Eng (2006). *Open yellow box indicates insertion sequence from intron

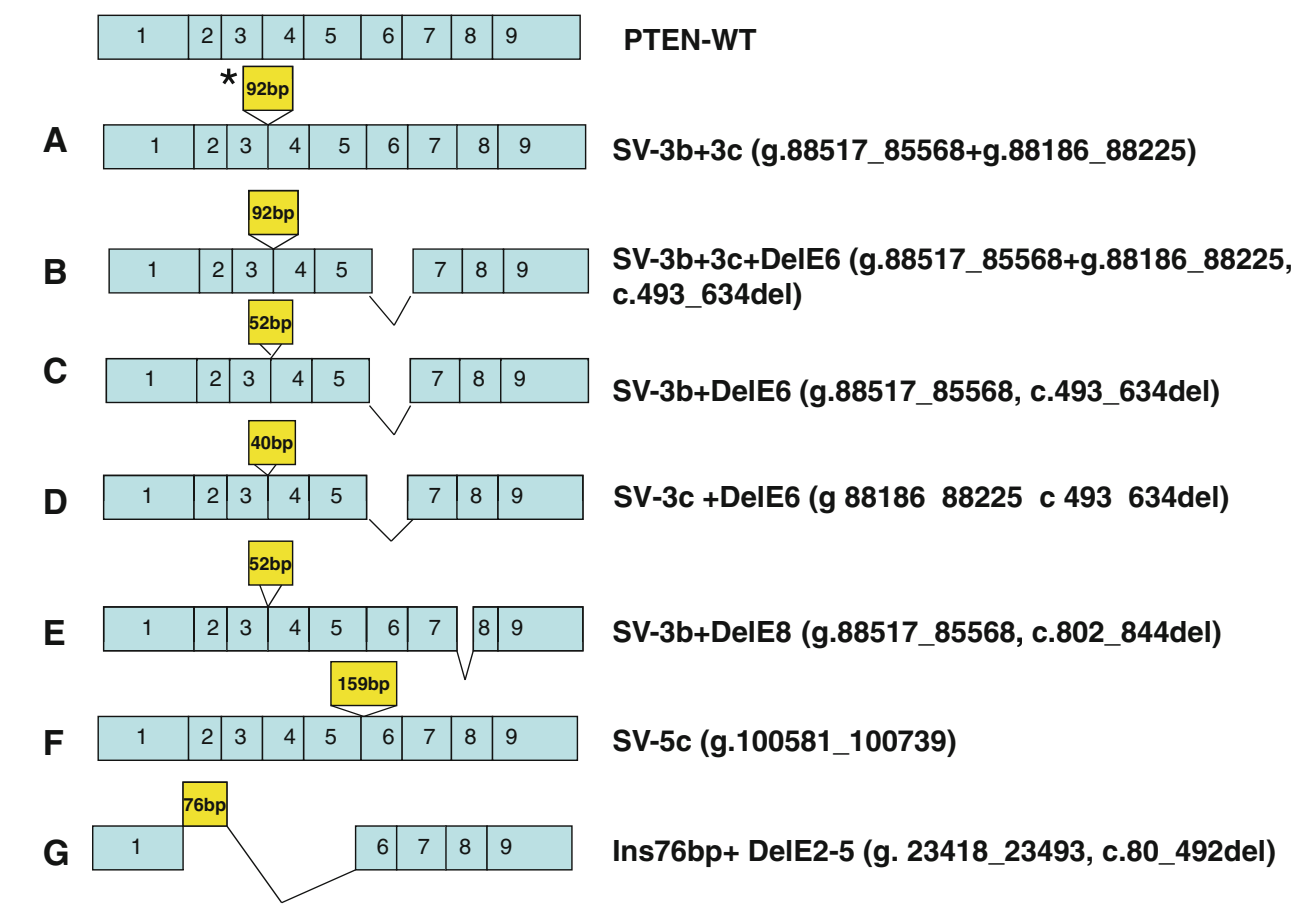

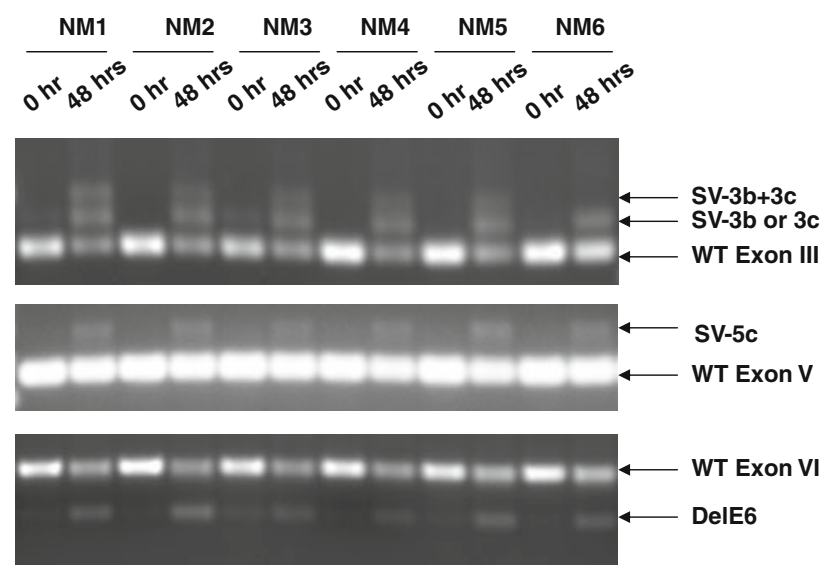

Fig. 2 Aberrant splicing of PTEN observed in "aged" blood. RNAs were extracted at the indicated time points. Genomic DNA was removed from RNAs using the DNA-free ${ }^{\mathrm{TM}}$ kit before revere-transcription. Primers for full length of PTEN-coding sequences are used in the first-run PCR, then a pair of exon-specific primers was used in the second run PCR for each exon and flanking region. $N M$ normal individual, $W T$ wild type, $S V$ splicing variant

PTEN SVs are found in both "fresh" and "aged" EBV-transformed lymphoblastoid cell lines from healthy individuals but have no affect on full-length PTEN protein expression

EBV-transformed lymphoblastoid cell lines are widely used for mutation screening. In order to investigate whether these SVs are present in EBV cell lines, we collected DNA and RNA from both "fresh" and 48-h "aged" EBV cell lines from six normal individuals. Although cells were treated with puromycin before extraction of RNA and preparation of cell lysates, SVs screening detected two types of SVs in both "fresh" and "aged" EBV-transformed lymphoblastoid cells from all six normal individual samples. They were SV-3c and DelE6 (Fig. 3a). No mutation was found in their DNA. The mechanism that causes alternative splicing in EBV-transformed cells is unclear. However, western blot data showed that the PTEN protein levels were not decreased in EBV lymphoblasts (Fig. 3b). Our data indicated that SVs can be detected in both "fresh" and "aged" EBV cell lines, but they had no impact on PTEN protein level.

\section{RNA integrity analysis}

In order to exclude that the aberrant splicing was the consequence of RNA degradation, RNA of MNCs from 12 normal individuals and EBV-transformed lymphoblastoid cell lines from 6 healthy individuals were analyzed for RIN. The RINs from "aged" blood were 7.5-9.0 (median 8.05), and significantly different from "fresh" blood (8.7-9.9, median 9.3, $p<0.01$ ) (Fig. 4a). Although this indicated that there was RNA degradation in "old" blood, but the RINs from "aged" EBV-transformed lymphoblastoid cells (8.8-10, median 9.8) had no significant difference from that of the "fresh" cells (8.9-10, median 10, $>>0.5$ ) (Fig. 4b), and the SVs were consistently found in both "fresh" and "aged" EBVtransformed cells. This indicated that the SVs were not associated with RNA degradation. In consideration that the primers that were used in the nested PCR and the first-run 


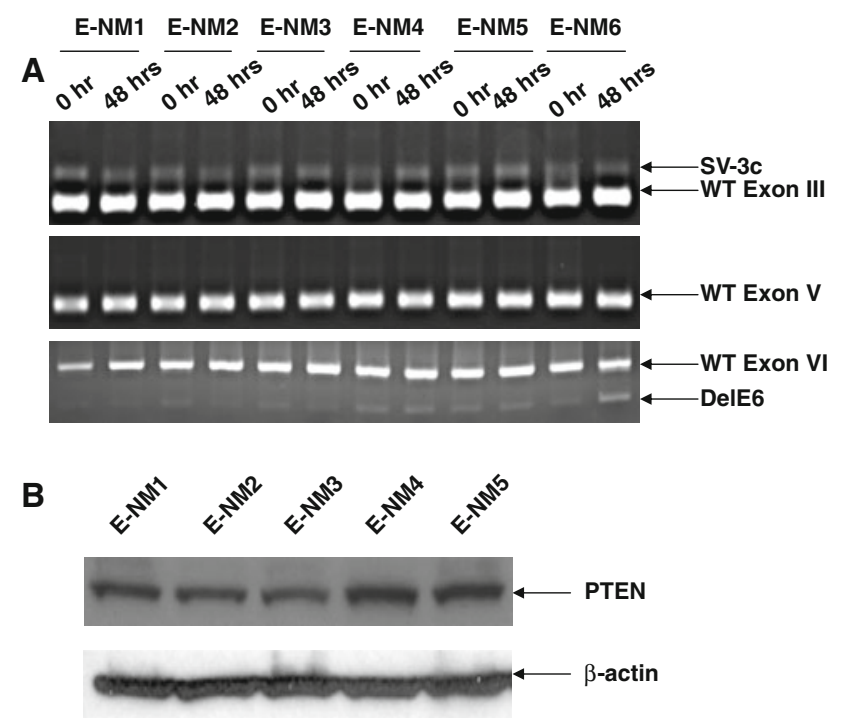

Fig. 3 Aberrant splicing of PTEN observed in "fresh" and "aged" EBV cell lines from normal individuals. a EBV lymphoblastoid cells from normal individuals were treated by puromycin $(150 \mu \mathrm{g} / \mathrm{ml})$ for $6 \mathrm{~h}$ before RNAs were extracted at the indicated time points. RT-PCR followed the protocol as described for MNCs from peripheral blood. b Normal PTEN protein expression in EBV cell lines with aberrant splicing of PTEN. Cell lysates were prepared from unstimulated EBV lymphoblastoid cells from normal individuals, which had been treated with puromycin $(150 \mu \mathrm{g} / \mathrm{ml})$ for $6 \mathrm{~h}$ before cell lysis. $E-N M$ represents EBV-transformed lymphoblasts from normal individual. WT wild type, $S V$ splicing variant

PCR for screening SVs would amplify only the full length of $P T E N$-coding sequence, and the most of the aberrant transcripts had intron retention, we are convinced that the observed aberrant transcripts were not caused by RNA degradation, instead, they were the consequence of relaxation of the splice fidelity when the samples were under stress.

\section{Discussion}

Somatic mutations that cause splicing deficits rarely occur. Germ-line sequence variations in both splice sites and regulatory elements have been implicated in susceptibility to cancer (Kalnina et al. 2005). Analysis of the cancer-related splicing-shifts suggests that many of these shifts act by disrupting a tumor suppressor gene (Xu and Lee 2003). However, some studies have demonstrated intriguing discontinuities between PTEN mutations and the subsequent phenotypes. Sequencing of cDNA from patients and normal individuals often reveals multiple SVs in PTEN, some of which are non-mutation related (Agrawal and Eng 2006). Agrawal and Eng (2006) reported that SVs, some of which were identical to the ones we found, were naturally occurring SVs in EBV cell lines, in patients with Cowden Syndrome, in sporadic breast cancer, and in a cDNA panel comprising different adult human tissues. They found
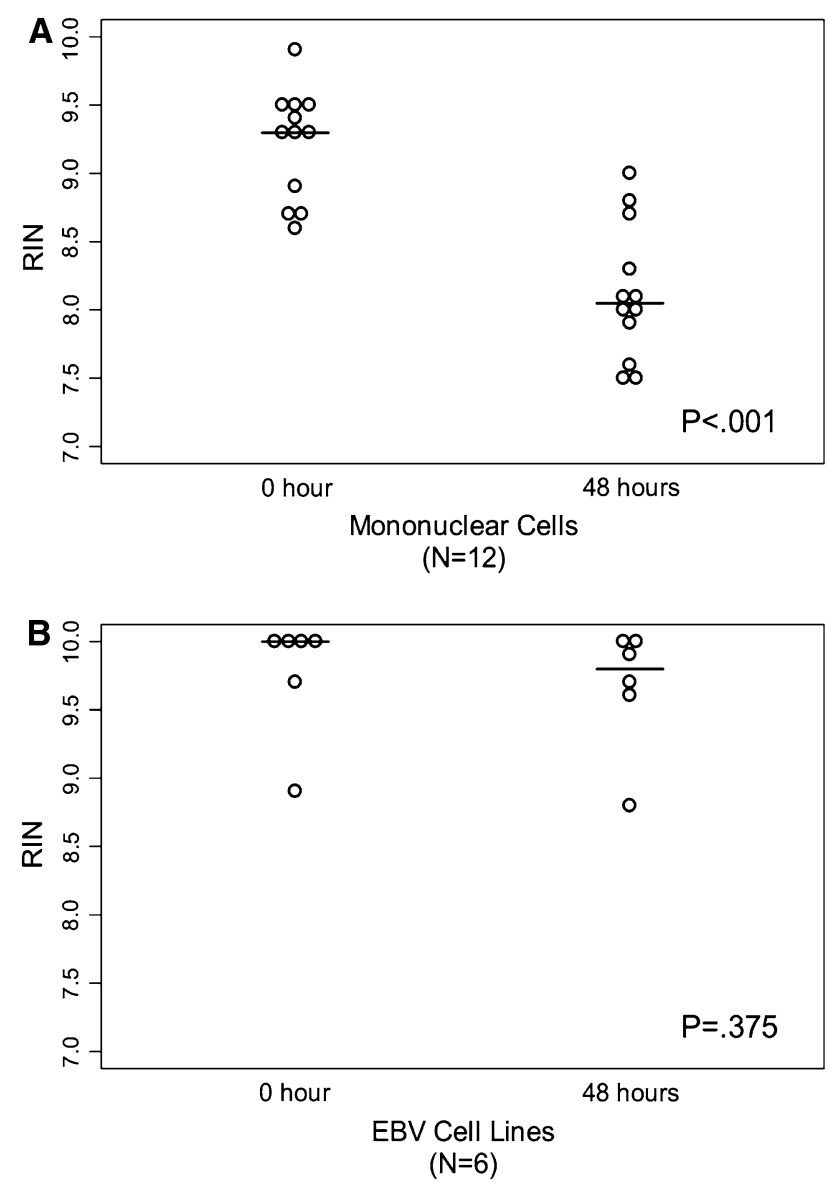

Fig. 4 Dot plots of RNA integrity number $(R I N)$ from "fresh" and "old" blood MNCs (a) and EBV lymphoblastoid cell Lines (b). The bars indicate the median levels of each group

SV-3b and $3 c$ were expressed inconsistently in normal breast tissue and breast cancer tissues. They also demonstrated that some of the SVs (SV-5c) had an impact on cyclin D1 promoter activity. Interestingly, they found that some of the SVs were regulated by over-expressed p53 in the MCF7 cell line.

Since most of the tumor suppressors are related to cell survival or death, it is rational to speculate that during the process of blood aging, while cells are coping with stress, many death-related mechanisms are initiated, which includes protein degradation and splicing alterations. This may cause the deregulation of protein and RNA, as well as abnormal DNA damage repair, which eventually leads to cell death or abnormal survival.

Illegitimate splicing is specifically referred to as the phenomenon of splice alterations that are due to the relaxation of the splice fidelity and does not naturally occur. Typically it is observed in aged samples, such as aged blood. It has been reported in other tumor suppressor genes, such as TSG101, FHIT, and NF1 by other groups (Gayther et al. 1997; Wimmer et al. 2000). It appears that this phenome- 
non is related to the genes that are linked to the function of cell survival. It is rational to speculate that illegitimate splicing might be a mechanism that cells utilize to cope with stress, which could function to either enhance or eliminate the accumulation of damaged cells or tissues. Our data indicates that the SVs of PTEN found in "aged" blood and EBV cell lines were caused by illegitimate splicing, not a pathological mechanism. Whether this mechanism applies to other tissues needs to be further investigated.

Many RNAs are tissue- and developmental-stagespecific. The awareness of the phenomenon of illegitimate splicing is becoming more important, especially in the era when RNA, and microRNA array studies are so popular. In addition to preventing possible false information that may be generated by amplification of pseudogenes, our study raises another issue that the age status of tissue should be addressed when we analyze and interpret RNA-related data.

In conclusion, we have demonstrated that illegitimate splicing occurs in the PTEN gene. This finding raises a novel issue regarding the sample handling in studies related to aberrant transcription. Published conclusions should be made with caution when data was generated from non-fresh samples, such as improperly preserved samples. When such data are reported, the procedure regarding how the samples and controls were handled should be addressed in detail.

Acknowledgments The authors would like to thank Dr. Ludwine M. Messiaen for general assistance in experiment design, Dr. Hyoungsoo Choi and Yuqing Liu for technical assistance, and Ms. Shelly Lensing for helping preparing Fig. 4. This work was supported in part by US Public Health Service grants R01 CA095621 and R01 HL082959 (P.D.E.) from the National Institutes of Health.

Conflict of interest The authors declare that they have no competing interests.

Open Access This article is distributed under the terms of the Creative Commons Attribution Noncommercial License which permits any noncommercial use, distribution, and reproduction in any medium, provided the original author(s) and source are credited.

\section{References}

Agrawal S, Eng C (2006) Differential expression of novel naturally occurring splice variants of PTEN and their functional consequences in Cowden syndrome and sporadic breast cancer. Hum Mol Genet 15:777-787

Caceres JF, Kornblihtt AR (2002) Alternative splicing: multiple control mechanisms and involvement in human disease. Trends Genet 18:186-193

Carracedo A, Pandolfi PP (2008) The PTEN-PI3K pathway: of feedbacks and cross-talks. Oncogene 27:5527-5541

Celebi JT, Wanner M, Ping XL, Zhang H, Peacocke M (2000) Association of splicing defects in PTEN leading to exon skipping or partial intron retention in Cowden syndrome. Hum Genet 107:234-238

Dahia PL, Marsh DJ, Zheng Z, Zedenius J, Komminoth P, Frisk T, Wallin G, Parsons R, Longy M, Larsson C, Eng C (1997) Somatic deletions and mutations in the Cowden disease gene, PTEN, in sporadic thyroid tumors. Cancer Res 57:4710-4713

Dahia PL, Aguiar RC, Alberta J, Kum JB, Caron S, Sill H, Marsh DJ, Ritz J, Freedman A, Stiles C, Eng C (1999) PTEN is inversely correlated with the cell survival factor Akt/PKB and is inactivated via multiple mechanisms in haematological malignancies. Hum Mol Genet 8:185-193

Gayther SA, Barski P, Batley SJ, Li L, de Foy KA, Cohen SN, Ponder BA, Caldas C (1997) Aberrant splicing of the TSG101 and FHIT genes occurs frequently in multiple malignancies and in normal tissues and mimics alterations previously described in tumours. Oncogene 15:2119-2126

Glick JL (1980) Fundamentals of human lymphoid cell culture, pp 103-107

Kalnina Z, Zayakin P, Silina K, Line A (2005) Alterations of premRNA splicing in cancer. Genes Chromosomes Cancer 42:342357

Kurose K, Zhou XP, Araki T, Cannistra SA, Maher ER, Eng C (2001) Frequent loss of PTEN expression is linked to elevated phosphorylated Akt levels, but not associated with p27 and cyclin D1 expression, in primary epithelial ovarian carcinomas. Am J Pathol 158:2097-2106

Liaw D, Marsh DJ, Li J, Dahia PL, Wang SI, Zheng Z, Bose S, Call KM, Tsou HC, Peacocke M, Eng C, Parsons R (1997) Germline mutations of the PTEN gene in Cowden disease, an inherited breast and thyroid cancer syndrome. Nat Genet 16:64-67

Liu YL, Castleberry RP, Emanuel PD (2009) PTEN deficiency is a common defect in juvenile myelomonocytic leukemia. Leuk Res 33:671-677

Marsh DJ, Coulon V, Lunetta KL, Rocca-Serra P, Dahia PL, Zheng Z, Liaw D, Caron S, Duboue B, Lin AY, Richardson AL, Bonnetblanc JM, Bressieux JM, Cabarrot-Moreau A, Chompret A, Demange L, Eeles RA, Yahanda AM, Fearon ER, Fricker JP, Gorlin RJ, Hodgson SV, Huson S, Lacombe D, Eng C et al (1998) Mutation spectrum and genotype-phenotype analyses in Cowden disease and Bannayan-Zonana syndrome, two hamartoma syndromes with germline PTEN mutation. Hum Mol Genet 7:507515

Sharrard RM, Maitland NJ (2000) Alternative splicing of the human PTEN/MMAC1/TEP1 gene. Biochim Biophys Acta 1494:282285

Soria JC, Lee HY, Lee JI, Wang L, Issa JP, Kemp BL, Liu DD, Kurie JM, Mao L, Khuri FR (2002) Lack of PTEN expression in nonsmall cell lung cancer could be related to promoter methylation. Clin Cancer Res 8:1178-1184

Tashiro H, Blazes MS, Wu R, Cho KR, Bose S, Wang SI, Li J, Parsons R, Ellenson LH (1997) Mutations in PTEN are frequent in endometrial carcinoma but rare in other common gynecological malignancies. Cancer Res 57:3935-3940

Wang SI, Puc J, Li J, Bruce JN, Cairns P, Sidransky D, Parsons R (1997) Somatic mutations of PTEN in glioblastoma multiforme. Cancer Res 57:4183-4186

Wimmer K, Eckart M, Rehder H, Fonatsch C (2000) Illegitimate splicing of the NF1 gene in healthy individuals mimics mutationinduced splicing alterations in NF1 patients. Hum Genet 106:311-313

Xu Q, Lee C (2003) Discovery of novel splice forms and functional analysis of cancer-specific alternative splicing in human expressed sequences. Nucleic Acids Res 31:5635-5643

Zhou XP, Waite KA, Pilarski R, Hampel H, Fernandez MJ, Bos C, Dasouki M, Feldman GL, Greenberg LA, Ivanovich J, Matloff E, Patterson A, Pierpont ME, Russo D, Nassif NT, Eng C (2003) Germline PTEN promoter mutations and deletions in Cowden/ Bannayan-Riley-Ruvalcaba syndrome result in aberrant PTEN protein and dysregulation of the phosphoinositol-3-kinase/Akt pathway. Am J Hum Genet 73:404-411 